\title{
A technical application of quantitative next generation sequencing for chimerism evaluation
}

\author{
MICHELANGELO ALOISIO $^{1 *}$, DANILO LICASTRO ${ }^{2 *}$, LUCIANA CAENAZZO $^{3}$, VALENTINA TORBOLI $^{1}$, \\ ANGELA D'EUSTACCHIO $^{4}$, GIOVANNI MARIA SEVERINI ${ }^{4 *}$ and EMMANOUILATHANASAKIS ${ }^{4}$ \\ ${ }^{1}$ Department of Life Sciences, University of Trieste, I-34127 Trieste; ${ }^{2}$ Cluster in Biomedicine, CBM S.c.r.l., \\ Bioinformatic Services, Area Science Park, I-34149 Basovizza; ${ }^{3}$ Department of Molecular Medicine, \\ University of Padova, I-35121 Padova; ${ }^{4}$ Department of Advanced Diagnostic and Clinical Trials, \\ Institute for Maternal and Child Health, IRCCS 'Burlo Garofolo', I-34137 Trieste, Italy
}

Received March 23, 2016; Accepted July 12, 2016

DOI: $10.3892 / \mathrm{mmr} .2016 .5593$

\begin{abstract}
At present, the most common genetic diagnostic method for chimerism evaluation following hematopoietic stem cell transplantation is microsatellite analysis by capillary electrophoresis. The main objective was to establish, through repeated analysis over time, if a complete chimerism was present, or if the mixed chimerism was stable, increasing or decreasing over time. Considering the recent introduction of next generation sequencing (NGS) in clinical diagnostics, a detailed study evaluating an NGS protocol was conducted, coupled with a custom bioinformatics pipeline, for chimerism quantification. Based on the technology of Ion AmpliSeq, a 44-amplicon custom chimerism panel was designed, and a custom bioinformatics pipeline dedicated to the genotyping and quantification of NGS data was coded. The custom chimerism panel allowed identification of an average of 16 informative recipient alleles. The limit of detection of the protocol was fixed at $1 \%$ due to the NGS background $(<1 \%)$. The protocol followed the standard Ion AmpliSeq library preparation and Ion Torrent Personal Genome Machine guidelines. Overall, the present study added to the scientific literature, identifying novel technical details for a possible future application of NGS for chimerism quantification.
\end{abstract}

Correspondence to: Dr Giovanni Maria Severini, Department of Advanced Diagnostic and Clinical Trials, Institute of Maternal and Child Health, IRCCS 'Burlo Garofolo', Via dell'Istria 65/1, I-34137 Trieste, Italy

E-mail: gianmaria.severini@burlo.trieste.it

${ }^{*}$ Contributed equally

Key words: chimerism, quantification, next generation sequencing, Ion Torrent, AmpliSeq, SNP

\section{Introduction}

Allogenic hematopoietic stem cell transplantation (HSCT) is the predominant treatment used to cure malignant and non-malignant hematological disorders. The number of HSCTs conducted has increased due to an overall improvement in the safety of the procedure resulting from reduced-intensity conditioning regimens, and the availability of new donor sources, available in the national registries $(1,2)$.

Reduced-intensity conditioning is widely used to avoid the complications of myeloablative conditioning to prepare for HSCT in the adult population. However, this procedure is associated with a high risk of complications that may result in graft loss. To prevent this, it is important to monitor chimerism for early intervention (3). Evaluation of chimerism status at regular intervals is useful to prevent risk of early graft rejection and relapse in patients suffering from malignant diseases. Quantification of the chimerism percentage is also a potential marker of minimal residual disease (MRD) for patients without suitable MRD markers and it provides useful information on graft vs. host disease and graft vs. tumor effects (4-6).

Chimerism analysis is a tool that allows to determination of the genotypic origin of post-transplantation hematopoiesis. Subsequent to HSCT, a patient presenting with $100.0 \%$ donor-origin cells during follow up is considered to have the status of complete chimerism (CC), patients in which the donor- and recipient-origin cells coexist have the status of mixed chimerism (MC) (7). Informative genetic markers are used to discriminate between recipient and donor genomes in order to detect the chimerism status (8).

At present, different approaches based on polymerase chain reaction (PCR) amplification of polymorphic DNA sequences (short tandem repeat, STR; single nucleotide polymorphism, SNP; and insertion/deletion, INDEL) are used for chimerism analysis. In the vast majority of laboratories, semi-quantitative fluorescent PCR of STRs is the procedure of choice for diagnostic purposes. The key advantage offered by this method is the highly polymorphic nature of the STR markers, which allow for a high probability of two-genome discrimination. Laboratories currently use different commercial multiplex kits for forensic identifications or in house assays, however 
have formed consortiums to standardize procedures and set guidelines for the correct interpretation of results, in order to improve this intrinsically semi-quantitative platform with a sensitivity of $1.0-3.0 \%(9)$.

Next generation sequencing (NGS) technologies are an innovation in human and animal genomics research, as they are capable of producing 100-fold more data than the most powerful Sanger based capillary sequencers; thus enabling researchers to investigate the large number of queries that remain to addressed (10).

NGS generates hundreds of giga-bases of nucleotide sequences per instrument run and produces this data at a lower cost, thus motivating researchers to use NGS for various purposes: To identify rare variations on the whole genome or on a target sequence, to analyze transcriptome profiling of cells, tissues and organisms and to identify epigenetic markers for disease diagnosis. Progress in the optimization of procedures, in addition to further reduction of costs, are the key factors that will lead to a more extensive uptake of this technique in diagnosis and for practical clinical applications.

NGS provides qualitative and quantitative data. Quantitative data depends on the depth of sequence data collected on each sample and on the quality of the target to expose. For samples with a lower abundance target, many more sequence reads are required to achieve accurate quantification (11). A previous study demonstrated that NGS exhibits sensitivity comparable to that of quantitative PCR (qPCR) in the evaluation of MRD in B cell disorders (12).

In the present study, an Ion AmpliSeq custom chimerism (ACCh) panel and a custom bioinformatic pipeline was created for chimerism quantification by NGS. The first aim was to detect the existence of cells of two origins in chimera samples and then to evaluate the capability of NGS to determine the percentage of the recipient cells.

\section{Materials and methods}

DNA sample preparation. The Ethics Committee of the Institute for Maternal and Child Health, IRCCS 'Burlo Garofolo' approved the present study (approval number: Prot. 18/2015, $\mathrm{Cl}$. M/11). Written informed consent was obtained from all the participants.

Total peripheral blood was collected from 10 volunteer donors (V01-V10; 4 males and 6 females) with ages ranging from 20-50 years, and from 2 pediatric patients that underwent allogeneic HSCT (pR1, male, 5 years; and pR2, male, 12 years) and their donors (pD1, male, 9 years; and $\mathrm{pD} 2$, female, 25 years; Table I). Written informed consent was obtained from all the participants.

All DNA samples were isolated using the QIAamp DNA Blood kit according to manufacturer's protocol (Qiagen $\mathrm{GmbH}$, Hilden, Germany). The DNA status was evaluated using the NanoDrop 1000 spectrophotometer (Thermo Fisher Scientific, Inc., Waltham, MA, USA) and the Qubit dsDNA HS Assay kit and the Qubit fluorometer (Thermo Fisher Scientific, Inc.). A DNA stock solution was prepared for all DNA samples at $20.0 \mathrm{ng} / \mu \mathrm{l}$.

DNA samples from the 10 volunteer donors were randomly paired. A total of 5 artificial chimeric DNA mixtures, as the donor/recipient chimera, were created by diluting DNA with its paired DNA at several percentages of the first DNA for each artificial DNA mixture $(\mathrm{aCh})$.

Ion AmpliSeq custom chimerism panel design. A multi phase strategy was employed to evaluate the main characteristics of the ACCh panel: i) The panel average heterozygosity was assessed around 0.5 for the European population (HapMap Phase 3 CEU population); ii) two SNPs per somatic chromosome, termed 'main SNPs' (mSNPs), were selected and located in two different regions of the same chromosome; iii) the amplicon composition was evaluated according to the following requirements: a) GC percentage ranging between 40.0 and $60.0 \%$; b) presence of one mSNP inside each amplicon; c) mSNP location preferably in the centre of the amplicon; d) absence of INDEL SNPs; e) absence of homopolymers and potential homopolymer generation from SNP variants and their flanking regions; f) absence of flanking SNPs to the mSNPs.

In total, 44 single-nucleotide, biallelic, polymorphisms were selected from the NCBI dbSNPs database (http://www.ncbi. nlm.nih.gov/SNP/, build 138, last database update 28.03.2014; Table II). A total of 4 base sequences including mSNPs were used as target regions for primer design. The primer pool, intended for DNA library construction through multiplex PCR, was defined by Ion AmpliSeq Designer software, version 3.0.1 (Thermo Fisher Scientific, Inc.). A single-tube, 44 primer pair pool was purchased from Life Technologies (Thermo Fisher Scientific, Inc.).

Ion torrent library preparation and sequencing. DNA sample library preparation was performed according to the AmpliSeq Library Preparation protocol (Life Technologies; Thermo Fisher Scientific, Inc.). For each DNA sample, a library was constructed using $10.0 \mathrm{ng}$ genomic DNA through the Ion AmpliSeq Library kit, version 2.0. Subsequently, according to the library preparation protocol, each DNA library was indexed using the Ion Xpress Barcode Adapters kit (Thermo Fisher Scientific, Inc.) and was purified using AMPure XP magnetic beads (Beckman Coulter, Inc., Brea, CA, USA). Each DNA library was then quantified by qPCR using the thermo-cycler 7900HT Fast Real-Time PCR system with the Ion Library TQMN Quantification kit (Thermo Fisher Scientific, Inc.). Template Ion Sphere Particles were arranged using the Ion Personal Genome Machine (PGM) Template OT2 200 kit (Thermo Fisher Scientific, Inc.) and a single end 200 base-read sequencing run was conducted using the Ion Torrent PGM system. Libraries were pooled at $8 \mathrm{pM}$ using the following rates: Donor/recipient, 1:1; and chimera/chimera, 1:1. The recipient/chimera rate was fixed at 1:40 in order to obtain an average coverage of the above libraries around 250X:10,000X. Library pools were sequenced on ion 314 and 316 chip (Table III).

Hotspot panel bed file. A hotspot panel bed file was created using the UCSC Genome Browser (https://genome.ucsc.edu). All SNPs located in the central region of each amplicon were included (NCBI dbSNPs build 138; 'Common SNPs' =286). All the INDELs present across the amplicons, and the SNPs near the $5^{\prime}$ and $3^{\prime}$ ends of the amplicons were excluded from the file. All the above 44 selected SNPs were marked as 'mSNP' 
Table I. List of the patient DNA samples used in the present study.

\begin{tabular}{|c|c|c|c|c|}
\hline \multicolumn{2}{|c|}{ DNA ID } & Chimera ID & Chimera information & Notes \\
\hline pD1 & $\mathrm{pR} 1$ & \multicolumn{2}{|c|}{ Samples used to evaluate panel informativity in consanguinity } & Brothers, pre-HSCT \\
\hline pD2 & $\mathrm{pR} 2$ & $\mathrm{pCh}_{1}$ & MC by STR-CE analysis ${ }^{\mathrm{a}}$ & +1 month post-HSCT \\
\hline pD2 & pR2 & $\mathrm{pCh}_{2}$ & CC by STR-CE analysis & +2 months post-HSCT \\
\hline pD2 & $\mathrm{pR} 2$ & $\mathrm{pCh}_{3}$ & MC by STR-CE analysis & +3 months post-HSCT \\
\hline $\mathrm{pD} 2$ & $\mathrm{pR} 2$ & $\mathrm{pCh}_{4}$ & MC by STR-CE analysis & +4 months post-HSCT \\
\hline pD2 & $\mathrm{pR} 2$ & $\mathrm{pCh}_{5}$ & MC by STR-CE analysis & +6 months post-HSCT \\
\hline pD2 & $\mathrm{pR} 2$ & $\mathrm{pCh}_{6}$ & CC by STR-CE analysis & +10 months post-HSCT \\
\hline
\end{tabular}

${ }^{\mathrm{a}}$ Chimerism evaluation of all patient samples $\left(\mathrm{pCh}_{1-6}\right)$ was performed by STR-CE analysis in an external laboratory (Department of Molecular Medicine, University of Padova, Padova, Italy). HSCT, hematopoietic stem cell transplantation; MC, mixed chimerism; CC, complete chimerism; STR-CE, short tandem repeat capillary electrophoresis.

and the SNPs belonging to the same amplicon were indexed with the same chromosome/amplicon ID number. Finally, a hotspot panel bed file was created: 'HP286SNPs'.

Genotyping and quantification. Genotyping of all DNA was performed automatically, together with the quantification of all the chimeras, using a custom bioinformatics tool. The code of our tool was written using the Shiny package in $\mathrm{R}$, a web framework to build interactive web applications (https://cran.r-project.org/web/packages/shiny/index.html). A functional diagram of the code is presented in Fig. 1 and the full code is available on request. The code is based on dependencies of the Bioconductor package that must be pre-installed for proper tool functionality.

The custom tool requires as input the sequencing bam files of 'Donor', ' Recipient' and 'Chimeric' patient. Briefly, it uses readGAlignments and pileLettersAt functions, from the GenomicAlignments package (13), to read bam files and extracts the letters/nucleotides into a set of individual genomic positions defined from the bed file. Thresholds for 'Donor' and 'Recipient', homozygous and heterozygous genotyping calls, are settled in base counts frequency ranges of 90.0-100.0\% and 30.0-60.0\%, respectively. Genotyping calls not included in the thresholds ranges were excluded as unreliable; users can modify the thresholds according to their needs from the user interface. Genotypes from each library were crosschecked to select only SNPs comparable in all conditions. Selected SNPs from donors and recipients were labelled as informative recipient alleles (IRA) according to the following schema: Donor homozygous and recipient heterozygous [Donor (AA) and Recipient (Aa); Donor (aa) and Recipient (Aa)]; donor and recipient homozygous for different alleles [Donor (AA) and Recipient (aa); Donor (aa) and Recipient (AA)].

Only the IRA SNPs tagged as informative were used to calculate the chimera's donor:recipient ratio as median of the allele frequency ratio, while standard error was used to calculate confidence intervals of prediction at $95.0 \%$.

To cross validate the tool, genotyping of all donor and recipient samples was also performed manually obtaining the variant data from the Ion Torrent plugin Variant Caller, version 4.4 using the 'Generic-PGM-Germ Line-Low Stringency' configuration coupled by the HP286SNPs hotspot bed file.

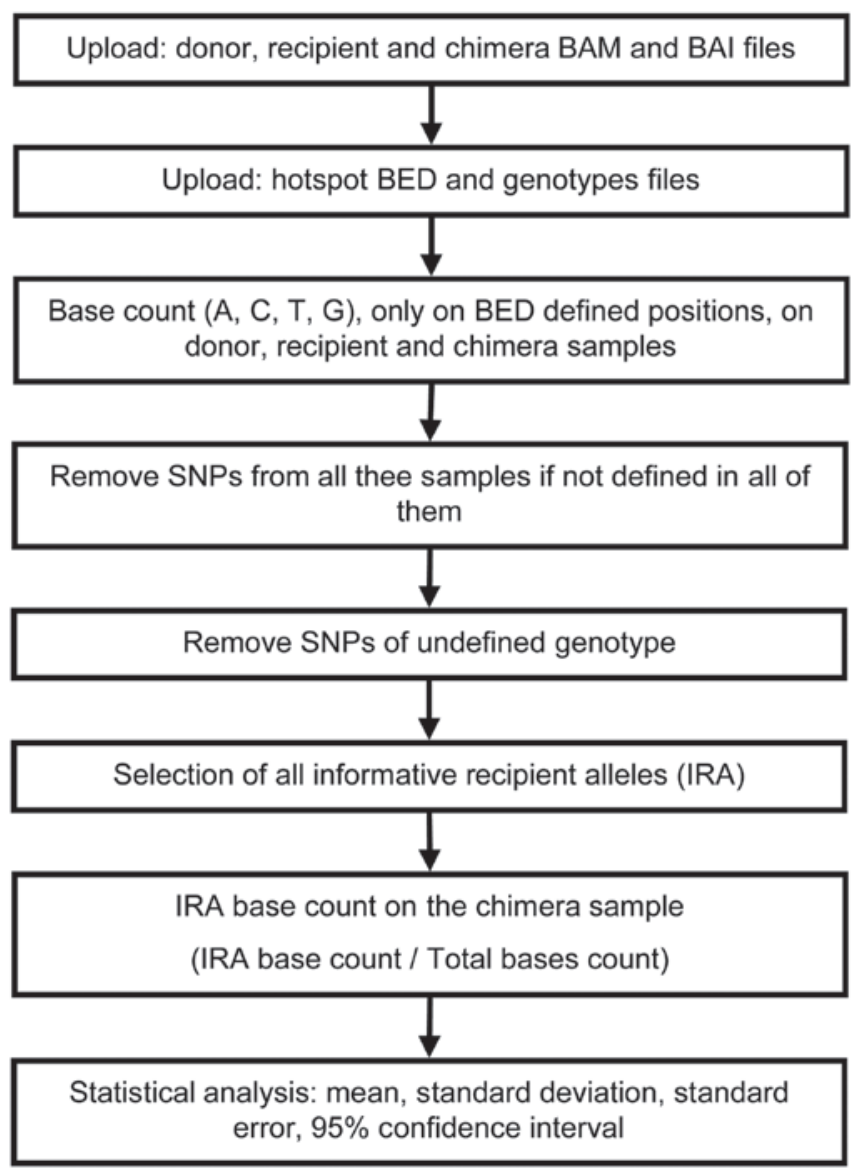

Figure 1. Code functional diagram. All output next generation sequencing files and reference files were uploaded to the custom pipeline. A quality control filtering step per target base was performed and the genotypes for donor and recipient samples were defined. Finally, subsequent to identification of the informative recipient allele, statistical analysis was conducted to determined the chimerism percentage. A, adenosine; $\mathrm{C}$, cytosine; $\mathrm{T}$, thymine; $\mathrm{G}$ guanine; SNP, single nucleotide polymorphism; IRA, informative recipient allele.

Microsatellite analysis and patient data validation. Multiplex PCR amplification of V01-V10 and $\mathrm{aCh}_{1-13}$ samples, in addition to the patient samples pD2, pR2 and $\mathrm{pCh}_{1-6}$, was performed according to the manufacturer's instructions of the 
Table II. List of all main SNPs included in the Ion AmpliSeq custom chimerism panel.

\begin{tabular}{|c|c|c|c|c|}
\hline SNP ID & Genome position & Alleles & $\begin{array}{c}\text { European } \\
\text { heterozygosity }\end{array}$ & $\begin{array}{l}\text { Informativity of } \\
\text { recipient allele } \%\end{array}$ \\
\hline rs12070036 & chr1:g.227819514 & $\mathrm{A} / \mathrm{G}$ & 0.407 & 41 \\
\hline rs1234315 & chr1:g.173178463 & $\mathrm{C} / \mathrm{T}$ & 0.513 & 37 \\
\hline rs10496711 & chr2:g.134516742 & $\mathrm{C} / \mathrm{G}$ & 0.407 & 40 \\
\hline rs12612347 & chr2:g.219057338 & $\mathrm{A} / \mathrm{G}$ & 0.442 & 40 \\
\hline rs1984630 & chr3:g.134414219 & $\mathrm{G} / \mathrm{T}$ & 0.522 & 36 \\
\hline rs9831477 & chr3:g.30693522 & $\mathrm{A} / \mathrm{T}$ & 0.483 & 38 \\
\hline rs10033900 & chr4:g.110659067 & $\mathrm{C} / \mathrm{T}$ & 0.496 & 37 \\
\hline rs5335 & chr4:g.148463840 & $\mathrm{C} / \mathrm{G}$ & 0.492 & 37 \\
\hline rs983889 & chr5:g.15555486 & $\mathrm{A} / \mathrm{C}$ & 0.487 & 38 \\
\hline rs10038113 & chr5:g.25902342 & $\mathrm{C} / \mathrm{T}$ & 0.469 & 38 \\
\hline rs552655 & chr6:g.13370488 & $\mathrm{A} / \mathrm{G}$ & 0.504 & 37 \\
\hline rs2077163 & chr6:g.33636907 & $\mathrm{C} / \mathrm{T}$ & 0.460 & 39 \\
\hline rs39395 & chr7:g.103489729 & $\mathrm{A} / \mathrm{G}$ & 0.425 & 40 \\
\hline rs2270188 & chr7:g.116140524 & $\mathrm{G} / \mathrm{T}$ & 0.496 & 38 \\
\hline rs10505477 & chr8:g.128407443 & $\mathrm{C} / \mathrm{T}$ & 0.531 & 36 \\
\hline rs532841 & chr8:g.12957475 & $\mathrm{C} / \mathrm{T}$ & 0.549 & 35 \\
\hline rs2297313 & chr9:g.91669362 & $\mathrm{A} / \mathrm{G}$ & 0.960 & 37 \\
\hline rs424539 & chr9:g.14442595 & $\mathrm{C} / \mathrm{G}$ & 0.467 & 38 \\
\hline rs 1561570 & chr10:g.13155726 & $\mathrm{C} / \mathrm{T}$ & 0.522 & 36 \\
\hline rs619824 & chr10:g.104581288 & $\mathrm{G} / \mathrm{T}$ & 0.407 & 41 \\
\hline rs198464 & chr11:g.61521621 & $\mathrm{C} / \mathrm{T}$ & 0.504 & 37 \\
\hline rs178503 & chr11:g.44082931 & $\mathrm{A} / \mathrm{G}$ & 0.442 & 40 \\
\hline rs1126758 & chr12:g.103248924 & $\mathrm{A} / \mathrm{G}$ & 0.416 & 40 \\
\hline rs8608 & chr12:g.53294381 & $\mathrm{A} / \mathrm{G}$ & 0.522 & 36 \\
\hline rs1061472 & chr13:g.52524488 & $\mathrm{A} / \mathrm{G}$ & 0.504 & 37 \\
\hline rs504544 & chr13:g.19735891 & $\mathrm{A} / \mathrm{T}$ & 0.508 & 37 \\
\hline rs10143250 & chr14:g.104723433 & $\mathrm{C} / \mathrm{T}$ & 0.434 & 39 \\
\hline rs1957779 & chr14:g.63669647 & $\mathrm{C} / \mathrm{T}$ & 0.449 & 39 \\
\hline rs634990 & chr15:g.35006073 & $\mathrm{A} / \mathrm{G}$ & 0.492 & 38 \\
\hline rs2117215 & chr15:g.94879684 & $\mathrm{C} / \mathrm{T}$ & 0.603 & 32 \\
\hline rs121893 & chr16:g.66183995 & $\mathrm{C} / \mathrm{T}$ & 0.414 & 39 \\
\hline rs 2191125 & chr16:g.7720923 & $\mathrm{T} / \mathrm{C}$ & 0.550 & 34 \\
\hline rs6808 & chr17:g.62400575 & $\mathrm{C} / \mathrm{G}$ & 0.450 & 39 \\
\hline rs744166 & chr17:g.40514201 & $\mathrm{T} / \mathrm{C}$ & 0.441 & 40 \\
\hline rs620898 & chr18:g.48509148 & $\mathrm{A} / \mathrm{T}$ & 0.467 & 38 \\
\hline rs633265 & chr18:g.57831468 & $\mathrm{A} / \mathrm{C}$ & 0.496 & 37 \\
\hline rs108295 & chr19:g.34224816 & $\mathrm{A} / \mathrm{G}$ & 0.496 & 37 \\
\hline rs892086 & chr19:g.10837677 & $\mathrm{C} / \mathrm{T}$ & 0.451 & 39 \\
\hline rs753381 & chr20:g.39797465 & $\mathrm{T} / \mathrm{C}$ & 0.451 & 39 \\
\hline rs715147 & chr20:g.50055350 & $\mathrm{G} / \mathrm{A}$ & 0.367 & 43 \\
\hline rs225436 & chr21:g.43729034 & $\mathrm{A} / \mathrm{G}$ & 0.517 & 36 \\
\hline rs8128316 & chr21:g.35721560 & $\mathrm{C} / \mathrm{T}$ & 0.542 & 35 \\
\hline rs4444 & chr22:g.31205334 & $\mathrm{A} / \mathrm{G}$ & 0.483 & 38 \\
\hline rs132985 & chr22:g.38563471 & $\mathrm{C} / \mathrm{T}$ & 0.517 & 37 \\
\hline
\end{tabular}

${ }^{a}$ In order to calculate the probability (informativity) to identify an informative recipient allele in a casual donor/recipient pair, the single values of probability of each genotype combination that carry an informative recipient allele were added as follows: Donor homozygous and recipient heterozygous [Donor (AA) and Recipient (Aa); Donor (aa) and Recipient (Aa)]; donor and recipient homozygous but for different alleles [Donor (AA) and Recipient (aa); Donor (aa) and Recipient (AA)]. SNP, single nucleotide polymorphism; A, adenine; G, guanine; C, cytosine; $\mathrm{T}$, thymine. 
Table III. List of the library pools sequenced by next generation sequencing.

\begin{tabular}{|c|c|c|c|}
\hline DNA ID & Chimera ID & Chimera notes & Ion chip \\
\hline V01, V02 & $\mathrm{aCh}_{1}$ & Chimera: $1.0 \%$ of V01; $99.0 \%$ of V02 & 314 \\
\hline V03, V04 & $\mathrm{aCh}_{2}$ & Chimera: $1.25 \%$ of V03; $98.75 \%$ of V04 & 314 \\
\hline V03, V04 & $\mathrm{aCh}_{3}$ & Chimera: $2.5 \%$ of V03; $97.5 \%$ of V04 & 314 \\
\hline V05, V06 & $\mathrm{aCh}_{4}$ & Chimera: $5.0 \%$ of V05; $95.0 \%$ of V06 & 314 \\
\hline V05, V06 & $\mathrm{aCh}_{5}$ & Chimera: $10.0 \%$ of V05; $90.0 \%$ of V06 & 314 \\
\hline V07, V08 & $\mathrm{aCh}_{6,7,8,9,10,11}$ & $\begin{array}{l}\text { Chimeras: } 0.5 \%, 1.0 \%, 4.0 \%, 8.0 \%, 12.0 \% \text {, } \\
20.0 \% \text { of } \mathrm{V} 07 ; 99.5 \%, 99.0 \%, 96.0 \%, 92.0 \% \text {, } \\
88 \%, 80.0 \% \text { of } \mathrm{V} 08\end{array}$ & 316 \\
\hline V09, V10 & $\mathrm{aCh}_{12,13}$ & $\begin{array}{l}\text { Chimeras: } 40.0 \% \text { of V09 and } 100 \% \text { of V09; } \\
60.0 \% \text { of V10 and } 0.0 \% \text { of V10 }\end{array}$ & 316 \\
\hline $\mathrm{pD} 2, \mathrm{pR} 2$ & $\mathrm{pCh}_{1,2,3,4,5,6}$ & Chimeras: $\mathrm{MC}, \mathrm{CC}, \mathrm{MC}, \mathrm{MC}, \mathrm{MC}, \mathrm{CC}$ of $\mathrm{pR} 2^{\mathrm{a}}$ & 316 \\
\hline $\mathrm{pD} 2, \mathrm{pR} 2$ & $\mathrm{pCh}_{2}$ & Technical replication & 314 \\
\hline pD1, pR1 & $\mathrm{pCh}_{6}$ & Technical replication & 314 \\
\hline
\end{tabular}

${ }^{a}$ All patient samples were previously analyzed by short tandem repeat capillary electrophoresis in an external laboratory (Department of Molecular Medicine, University of Padova, Padova, Italy).

AmpFISTR Identifiler Plus PCR Amplification kit (Thermo Fisher Scientific, Inc.). Amplicons were resolved on a Genetic Analyzer 3130 and analyzed with GeneMapper software, version 4.1 (Life Technologies; Thermo Fisher Scientific, Inc.).

Patient samples $\left(\mathrm{pCh}_{1-6}\right)$ were also analyzed by qPCR (data not shown), as previously investigated by Bai et al (14). This analysis was performed as an additional validation method of NGS data, where a discrepancy between NGS and STR data was present.

\section{Results}

Ion chips and ACCh panel performance. A total of 7 library pools were loaded and sequenced on ion chip 314. Each pool was comprised of one donor, one recipient and one chimera. In addition, three additional pools were run on ion 316 chip (Table III). The mean values of performance of the 10 runs and of all samples are summarized in Table IV.

NGS genotyping performances using the ACCh panel. A total of 14 DNA samples were genotyped on Ion Torrent PGM using the ACCh panel with the HP286SNPs bed file. Regarding the mSNPs, the Variant Caller output identified that $2 \mathrm{mSNPs}$ (rs121893 and rs12612347) were assigned as 'No Call' in over $50.0 \%$ of the genotyping runs due to low quality. The remaining $42 \mathrm{mSNPs}$ were successfully genotyped. Concerning the remaining 242 SNPs, 27 SNPs were assigned as 'No Call', with an average of 10 SNPs per patient. The call of these SNPs failed in two Variant Caller filtering steps: 'Maximum common signal shift' and 'minimum coverage on either strand'.

Genotyping of all samples was additionally performed using our tool with the HP286SNPs bed file (Table V). To identify the IRAs, the data of the donor was compared with the recipient using the tool and manually cross-validated with the Variant Caller genotypes. Inside the genotyping calls of

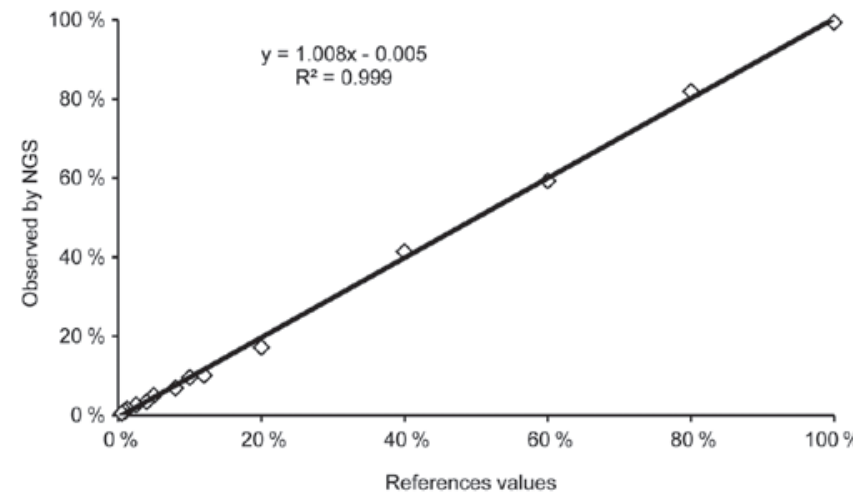

Figure 2. Linearity of Ion AmpliSeq custom chimerism panel: NGS against references values. A series of artificial chimeras, ranging between $0.5-100.0 \%$, were quantified by NGS and plotted against their reference values. NGS, next generation sequencing.

242 SNPs, a small bias was present between these 2 tools; this is due to the high conserved filters of variant caller, dedicated predominantly for standard sequencing applications, and due to the absence of these filters in our custom tool.

NGS linearity, detection limit and accuracy with the ACCh panel. In order to test the linearity of Ion Torrent PGM with the ACCh panel in a fixed detection range (0.5-100.0\%), a series of DNA mixtures was developed, diluting a DNA with its paired DNA at several percentages of the original. In order to increase the genetic marker variability in addition to the biological variability, a total of 12 artificial chimeras $\left(\mathrm{aCh}_{1-12}\right)$ were prepared from 5 different DNA pairs. Finally a pure DNA (V09) was run as $100.0 \%$ DNA $\left(\mathrm{aCh}_{13}\right)$. Subsequent to Ion Torrent sequencing, using the custom tool, quantitative data for all IRAs of each artificial chimera were obtained.

In addition, to increase the putative points in the dynamic range, the informative alleles of both DNA in the chimeras 
Table IV. In-house Ion Torrent Personal Genome Machine analysis observed average performances using the Ion AmpliSeq custom chimerism panel on ion 314 and 316 chips.

\begin{tabular}{lcc}
\hline A, In-house observed performance & Ion 314 chip & Ion 316 chip \\
\hline Ion sphere particles loading & $84.3 \%$ & $70.0 \%$ \\
Total bases (Mb) & 105.1 & 677.0 \\
Total reads & 533,535 & $3,474,065$ \\
Reads on-target & $99.7 \%$ & $99.3 \%$ \\
Panel uniformity & $97.6 \%$ & $96.0 \%$ \\
\hline & & Ion 316 chip \\
B, Coverage performance & Ion 314 chip & $43 / 44$ \\
Chimera samples - amplicons over 2,500X & $43 / 44$ & (range 4,777X-28,314X) \\
& range 4,069X-23,944X) & $44 / 44$ \\
Donor \& recipient samples - amplicons over 50X & (range 105X-952X) & (range 75X-2328X)
\end{tabular}

Table V. List of the number of IRAs identified in each DNA pair using the custom pipeline.

\begin{tabular}{|c|c|c|c|c|c|}
\hline \multirow{2}{*}{$\begin{array}{l}\text { DNA pair } \\
\text { 'Donor'/'Recipient' }\end{array}$} & \multicolumn{2}{|c|}{ Custom pipeline } & \multicolumn{2}{|c|}{ IRA genotypes } & \multirow{2}{*}{$\begin{array}{l}\text { Total IRAs } \\
\qquad(\%)^{\mathrm{a}}\end{array}$} \\
\hline & 44 mSNPs & 242 SNPs & Heterozygous & Homozygous & \\
\hline V01/V02 & 15 & 3 & 10 & 8 & $18(43)$ \\
\hline V03/V04 & 18 & 4 & 19 & 3 & $22(52)$ \\
\hline V05/V06 & 14 & 4 & 14 & 4 & $18(43)$ \\
\hline V07/V08 & 13 & 5 & 10 & 8 & $18(43)$ \\
\hline V09/V10 & 8 & 3 & 8 & 3 & $11(26)$ \\
\hline V02/V01 & 15 & 5 & 12 & 8 & $20(48)$ \\
\hline V04/V03 & 7 & 2 & 6 & 3 & $9(21)$ \\
\hline V06/V05 & 14 & 2 & 12 & 4 & $16(38)$ \\
\hline V08/V07 & 16 & 4 & 12 & 8 & $20(48)$ \\
\hline V10/V09 & 19 & 3 & 19 & 3 & $22(52)$ \\
\hline $\mathrm{pD} 1 / \mathrm{pR} 1$ & 9 & 2 & 9 & 2 & $11(26)$ \\
\hline $\mathrm{pD} 2 / \mathrm{pR} 2$ & 17 & 4 & 18 & 3 & $21(50)$ \\
\hline
\end{tabular}

${ }^{a}$ The percentage of each IRA was calculated on $42-44$ amplicons, due to the low quality of the remaining two, considering that each amplicon could contain at least one potential IRA. IRA, informative recipient allele; mSNP, main single nucleotide polymorphism.

$\mathrm{aCh}_{12}(40.0 \%$ of $\mathrm{V} 09$ and $60.0 \%$ of $\mathrm{V} 10)$ and $\mathrm{aCh}_{11}(20.0 \%$ of V07 and $80.0 \%$ of V08) were calculated and quantified.

Least-squares analysis of the above putative points identified a clear linearity $\left(\mathrm{R}^{2}=0.999 ; \mathrm{Y}=1.008 \mathrm{X}-0.005\right)$ between NGS and the reference values (Fig. 2).

Analyzing the artificial chimeras $\mathrm{aCh}_{6-13}$ by capillary electrophoresis using the STRs markers, chimerism ranging from 4.0-100.0\% was detected. Least-squares analysis identified a clear linearity $\left(\mathrm{R}^{2}=0.999 ; \mathrm{Y}=1.012 \mathrm{X}-0.009\right)$ between NGS and STRs values (Fig. 3).

In addition, the background of Ion Torrent generated by the ACCh panel was estimated. In this case, the custom tool was used, considering the 'donor' samples as chimera. The average background value at each SNP was estimated at $0.3 \%$ (range, $0.0-0.8 \%$ ) and with a $95.0 \%$ confidence interval between 0.1 and $0.5 \%$. Considering the background values and the reported literature on the error rates at each base of NGS technologies (range from 0.04-1.0\%) (15), the detection limit of the NGS protocol with the ACCh panel was set at $1.0 \%$, although an artificial chimera was detected at $0.5 \%$.

Finally, considering that the method determined each chimera, calculating the average value of all IRA, the average standard error was used as an indirect marker of accuracy, using the data of all artificial chimeras ranging from $1.0-99.0 \%$ (excluding 0.5 and $100.0 \%$ ). For the dynamic range of $1.0-20.0 \%$ of chimeras, the average standard error was 


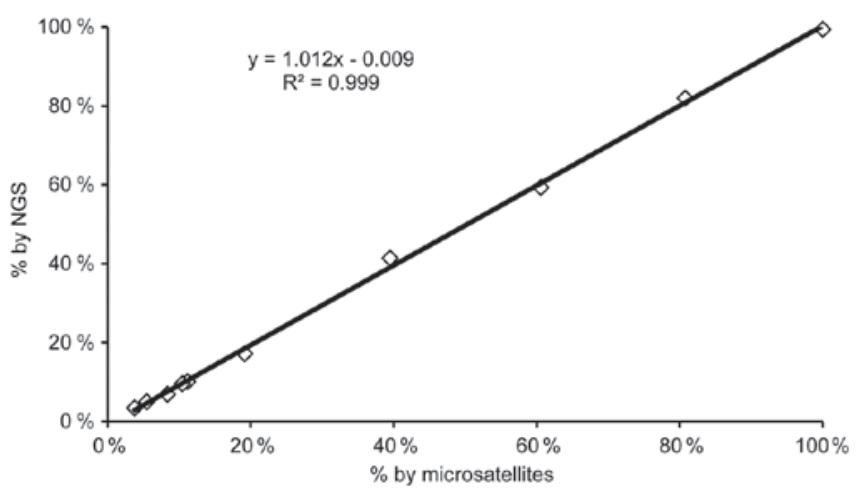

Figure 3. Linearity of Ion AmpliSeq custom chimerism panel: NGS against microsatellite values. A series of artificial chimeras were quantified by a standard microsatellite method (short tandem repeats) and the data were plotted compared to the NGS values. The chimeras ranging between $0.5-2.5 \%$ were not able to be quantified by short tandem repeats due to the detection limit of the informative markers. NGS, next generation sequencing.

calculated at $0.3 \%$ with a deviation at $0.2 \%$. For higher values of chimeras, up to $99.0 \%$, the standard error increased up to $1.8 \%$ with a maximum deviation at $2.0 \%$.

Patient chimerism evaluation on the NGS platform. Considering the linearity between NGS and reference values and between NGS and STR values using the standard NGS workflow and the custom tool, our workflow was tested in 6 samples of the same patient $\left(\mathrm{pCh}_{1-6}\right)$ in which the chimerism quantification report, previously performed by microsatellite analysis at different times in an external laboratory (Department of Molecular Medicine, University of Padova, Padova, Italy), presented at least one $\mathrm{CC}$ between two MCs (Table I).

NGS analysis detected a mixed chimerism in all samples $\left(\mathrm{pCh}_{1}, \mathrm{pCh}_{2}, \mathrm{pCh}_{3}, \mathrm{pCh}_{4}, \mathrm{pCh}_{5}\right.$ and $\left.\mathrm{pCh}_{6}\right)$ while the microsatellite analysis only in 4 of them $\left(\mathrm{pCh}_{1}, \mathrm{pCh}_{3}, \mathrm{pCh}_{4}\right.$ and $\left.\mathrm{pCh}_{5}\right)$. For these $4 \mathrm{MC}$ samples, the percentage of predicted chimerism was equal between the 2 methods of analysis (Fig. 4). In regard to the $\mathrm{pCh}_{2}$ and $\mathrm{pCh}_{6}$ samples, NGS analysis evaluated a mixed chimerism at 3.0 and $2.0 \%$, respectively (Fig. 4).

To confirm the obtained NGS data for the $\mathrm{pCh}_{2}$ and $\mathrm{pCh}_{6}$ samples, the NGS analysis was repeated, and qPCR was additionally perfomed in all patient samples. The results of qPCR analysis were in agreement with that of the NGS and microsatellite data for the $\mathrm{pCh}_{1}, \mathrm{pCh}_{3}, \mathrm{pCh}_{4}$ and $\mathrm{pCh}_{5}$, and confirmed the NGS data for the $\mathrm{pCh}_{2}$ and $\mathrm{pCh}_{6}$ samples.

\section{Discussion}

NGS technologies have revolutionized the field of genomics, and its application has been extended to different fields such as clinical diagnostics and forensic science (15-17). As a result of the continuous development of NGS, several applications previously performed on Sanger sequencing with capillary electrophoresis have been transferred onto the NGS platform, enabling fast and cost-effective generation sequence data with high resolution and accuracy. For this reason, different panels are being developed for the sequencing of genetic mutations involved in human diseases (e.g. MiSeqDx Cystic Fibrosis Clinical Sequencing Assay; Illumina, Inc.,

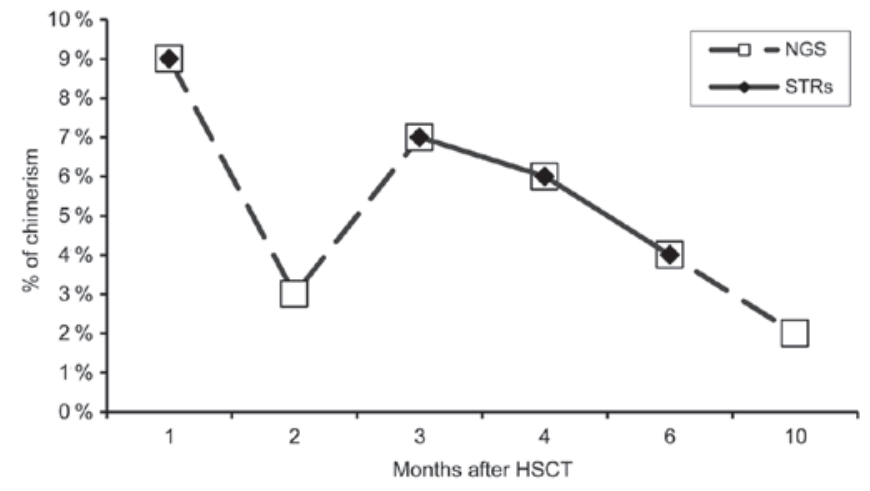

Figure 4. Patient sample evaluation with NGS and microsatellites (STRs) methods. Samples $(n=6)$ taken from the same patient at different time points, ranging from 1-10 months subsequent to HSCT, were compared using the two methods. At $+2\left(\mathrm{pCh}_{2}\right)$ and $+10\left(\mathrm{pCh}_{6}\right)$ months, the STRs method was not able to detect the recipient DNA. NGS, next generation sequencing; STRs, short tandem repeats; HSCT, hematopoietic stem cell transplantation.

San Diego, CA, USA) or in cancer (Ion AmpliSeq BRCA1 and BRCA2 Panel; Life Technologies; Thermo Fisher Scientific, Inc.).

In the field of chimerism quantification by NGS platforms, Debeljak et al (18) reported an innovative and well performed study, using haplotype counting. In addition, Kim et al (19) briefly reported a relative quantification analysis of SNP markers by NGS in one human bone marrow chimerism sample. However, the study by Kim et al (19) was conducted in a $4.9 \%$ chimerism sample without any detail concerning the limit of detection, the technical error or additional important technical information and validation data of NGS application in chimerism quantification.

In the present study, a full workflow was designed, and the proposed protocols and a bioinformatics tool were tested for chimerism quantification by NGS. A 44-amplicon custom chimerism panel based on Ion AmpliSeq technology was designed, and in addition a bioinformatics tool dedicated to the genotyping and quantification of NGS data was coded. These resources were created in order to provide a novel tool for the evaluation of the chimerism following allogenic HSCT, thus potentially increasing the number of clinical analyses supported on NGS platforms.

The ACCh proposed panel is composed of 44 amplicons, containing 44 selected mSNPs, of which 2 mSNPs are located in different regions of each somatic chromosome. It is suggested that the different mSNP locations in all somatic chromosome may be useful to avoid predominantly false negatives results caused by chromosomal deletions characteristic of certain malignancies (20). In addition, the bed file uploaded in the custom tool, containing all targeted SNPs, can be modified in order to exclude the SNPs present in chromosome target regions subjected to deletions in a specific patient.

The panel average heterozygosity was assessed around 0.5 for the European population in order to obtain different informative markers for each transplanting pair, for a more precise and robust quantification. The theoretical panel informativity for unrelated donor:recipient values, calculated according to the data present on the NCBI dbSNPs database, was estimated to be approximately $16 / 42 \mathrm{mSNPs}$, while for siblings the informativity 
was estimated at $50.0 \%$ (approximately $8 / 42 \mathrm{mSNPs}$ ). In order to increase the informativity of the ACCh panel, an additional 242 selected SNPs present in the targeted regions were included in the bed file. This addition of SNPs experimentally increased the average informativity (Table V).

The ACCh panel reached the limit of detection on the Ion Torrent PGM platform of $0.5 \%$, however, this was updated to the conservative value of $1.0 \%$ for two reasons: i) The Ion Torrent error is defined to be between 0.04 and $1.0 \%$ (21); and ii) the background of the ACCh panel, based on the IRA data of our experiments, ranges between 0.1 and $0.5 \%$.

Regarding the timing of chimerism analysis, the UK NEQAS Consortium has recommended that results should be assessed in 5 working days from the reception of the sample and in 3 working days for urgent requests (6). The protocol suggested in the current study is feasible in 2 days; the first day for library preparation and quantification and the second for template preparation, run sequence and data analysis. In addition, due to the fact the ACCh protocol suggested in the current study does not present any differences from the standard AmpliSeq Library Preparation and Ion Torrent PGM Run Sequence protocols, it is possible to introduce it to a standard manual library preparation workflow or in a library preparation workstation.

Considering the AmpliSeq library construction protocol (based on multiplex PCR) from Life Technologies (Thermo Fisher Scientific, Inc.), the same concept could be tested on additional NGS platforms, such as Illumina or Roche, according to their library preparation protocols. Concerning the custom bioinformatics tool, any bam and bai file coupled by a bed file, generated from any platform could be used.

At present, the cost of NGS analysis, compared with microsatellite methods, remains high, however considering the continuously reducing cost per NGS run, an NGS-based method for chimerism quantification could be evaluated in the future for its adoption in laboratories with a high volume of activity, and with NGS platforms already in use for other purposes. Although the present study reported a clear correlation between NGS and STR methods and identifed important technical details, further experimental replications are required in order for the NGS protocol to be validated for future laboratory use.

\section{Acknowledgements}

The current study was supported by a grant from the Institute of Maternal and Child Health-IRCCS 'Burlo Garofolo' (grant no. RC 16-2014). The authors would like to thank Dr Erika Ferrari for assistance with SNP data crosschecking.

\section{References}

1. Gratwohl A, Baldomero H, Gratwohl M, Aljurf M, Bouzas LF, Horowitz M, Kodera Y, Lipton J, Iida M, Pasquini MC, et al: Quantitative and qualitative differences in use and trends of hematopoietic stem cell transplantation: A Global Observational Study. Haematologica 98: 1282-1290, 2013.

2. Passweg JR, Baldomero H, Gratwohl A, Bregni M, Cesaro S, Dreger P, de Witte T, Farge-Bancel D, Gaspar B, Marsh J, et al: The EBMT activity survey: 1990-2010. Bone Marrow Transplant 47: 906-923, 2012.

3. Oshrine BR, Olson TS and Bunin N: Mixed chimerism and graft loss in pediatric recipients of an alemtuzumab-based reduced-intensity conditioning regimen for non-malignant disease. Pediatr Blood Cancer 61: 1852-1859, 2014.
4. Lawler M, McCann SR, Marsh JC, Ljungman P, Hows J, Vandenberghe E, O'Riordan J, Locasciulli A, Socié G, Kelly A, et al: Serial chimerism analyses indicate that mixed haemopoietic chimerism influences the probability of graft rejection and disease recurrence following allogeneic stem cell transplantation (SCT) for severe aplastic anaemia (SAA): Indication for routine assessment of chimerism post SCT for SAA. Br J Haematol 144: 933-945, 2009.

5. Terwey TH, Hemmati PG, Nagy M, Pfeifer H, Gökbuget N, Brüggemann M, Le Duc TM, le Coutre P, Dörken B and Arnold R: Comparison of chimerism and minimal residual disease monitoring for relapse prediction after allogeneic stem cell transplantation for adult acute lymphoblastic leukemia. Biol Blood Marrow Transplant 20: 1522-1529, 2014.

6. Clark JR, Scott SD, Jack AL, Lee H, Mason J, Carter GI, Pearce L, Jackson T, Clouston H, Sproul A, et al: Monitoring of chimerism following allogeneic haematopoietic stem cell transplantation (HSCT): Technical recommendations for the use of short tandem repeat (STR) based techniques, on behalf of the United Kingdom national external quality assessment service for leucocyte immunophenotyping chimerism working group. Br J Haematol 168: 26-37, 2015.

7. Bader P, Kreyenberg H, Hoelle W, Dueckers G, Handgretinger R, Lang P, Kremens B, Dilloo D, Sykora KW, Schrappe M, et al: Increasing mixed chimerism is an important prognostic factor for unfavorable outcome in children with acute lymphoblastic leukemia after allogeneic stem-cell transplantation: Possible role for pre-emptive immunotherapy? J Clin Oncol 22: 1696-1705, 2004.

8. Gineikiene E, Stoskus M and Griskevicius L: Single nucleotide polymorphism-based system improves the applicability of quantitative PCR for chimerism monitoring. J Mol Diagn 11: 66-74, 2009.

9. Lion T, Watzinger F, Preuner S, Kreyenberg H, Tilanus M, de Weger R, van Loon J, de Vries L, Cavé H, Acquaviva C, et al: The EuroChimerism concept for a standardized approach to chimerism analysis after allogeneic stem cell transplantation. Leukemia 26: 1821-1828, 2012.

10. Pareek CS, Smoczynski R and Tretyn A: Sequencing technologies and genome sequencing. J Appl Genet 52: 413-435, 2011.

11. Xuan J, Yu Y, Qing T, Guo L and Shi L: Next-generation sequencing in the clinic: Promises and challenges. Cancer Lett 340: 284-295, 2013.

12. Ladetto M, Brüggemann M, Monitillo L, Ferrero S, Pepin F, Drandi D, Barbero D, Palumbo A, Passera R, Boccadoro M, et al: Next-generation sequencing and real-time quantitative PCR for minimal residual disease detection in B-cell disorders. Leukemia 28: 1299-1307, 2014.

13. Lawrence $M$, Huber W, Pagès $H$, Aboyoun $P$, Carlson $M$, Gentleman R, Morgan MT and Carey VJ: Software for computing and annotating genomic ranges. PLoS Comput Biol 9: e1003118, 2013.

14. Bai L, Deng YM, Dodds AJ, Milliken S, Moore J and Ma DD: A SYBR green-based real-time PCR method for detection of haemopoietic chimerism in allogeneic haemopoietic stem cell transplant recipients. Eur J Haematol 77: 425-431, 2006.

15. Mwaigwisya S, Assiri RA and O'Grady J: Emerging commercial molecular tests for the diagnosis of bloodstream infection. Expert Rev Mol Diagn 15: 681-692, 2015.

16. D'Argenio V, Esposito MV, Telese A, Precone V, Starnone F, Nunziato M, Cantiello P, Iorio M, Evangelista E, D'Aiuto M, et al: The molecular analysis of BRCA1 and BRCA2: Next-generation sequencing supersedes conventional approaches. Clin Chim Acta 446: 221-225, 2015.

17. Børsting $\mathrm{C}$ and Morling $\mathrm{N}$ : Next generation sequencing and its applications in forensic genetics. Forensic Sci Int Genet 18: 78-89, 2015.

18. Debeljak M, Freed DN, Welch AJ, Haley L, Beierl K, Iglehart BS, Pallavajjala A, Gocke CD, Leffell MS, Lin MT, et al: Haplotype counting by next-generation sequencing for ultrasensitive human DNA detection. J Mol Diagn 16: 495-503, 2014.

19. Kim J, Hwang IS, Kim HS, Joo DJ, Hong KR and Choi JR: Bone marrow chimerism detection using next generation sequencing based on single nucleotide polymorphisms following liver transplantation: Comparison with short tandem repeat-PCR. Ann Lab Med 36: 82-84, 2016.

20. Chen DP, Tsai SH, Tseng CP, Wu TL, Chang PY and Sun CF: Bone marrow transplant relapse with loss of an allele. Clin Chim Acta 387: 161-164, 2008.

21. Bragg LM, Stone G, Butler MK, Hugenholtz P and Tyson GW: Shining a light on dark sequencing: Characterizing errors in Ion Torrent PGM data. PLoS Comput Biol 9: e1003031, 2013. 